7 2. Molecular and Cellular Biology Program, University of Washington, Seattle, WA, USA.

8 3. Institute for Stem Cells and Regenerative Medicine, University of Washington, Box 358056,

9 Seattle, WA, 98109, USA.

10 4. Department of Biological and Vision Sciences, The State University of New York, College of 11 Optometry, New York, NY, USA 


\section{Cis-Regulatory Accessibility Directs Muller Glial Development and Regenerative Capacity}

17 Leah S. VandenBosch ${ }^{1,2}$, Stefanie G. Wohl ${ }^{1}$, Matthew S. Wilken ${ }^{1,2}$, Kristen Cox ${ }^{1}$, Laura

Chipman ${ }^{1}$, Thomas A. Reh ${ }^{1,3 *}$

\section{Abstract}

Diseases and damage to the retina lead to losses in retinal neurons and eventual visual impairment. Although the mammalian retina has no inherent regenerative capabilities, fish have robust regeneration from Müller glia (MG). Recently, we have shown that driving expression of Ascl1 in adult mouse MG stimulates neurogenesis similar to fish regeneration. The regeneration observed in the mouse is limited in the variety of neurons that can be derived from MG; Ascl1expressing MG primarily generate bipolar cells. To better understand the limits of MG-based regeneration in mouse retinas, we used ATAC- and RNA-seq to compare newborn progenitors with MG. Our analysis demonstrated striking similarities between MG and progenitors, with losses in regulatory motifs for neurogenesis genes. Young MG were found to have intermediate expression profiles and accessible DNA, which is mirrored in the ability of Ascl1 to direct bipolar neurogenesis in young MG. When comparing what makes bipolar and photoreceptor cells distinct from glial cells, we find that bipolar-specific accessible regions are more frequently linked to bHLH motifs and Ascl1 binding, indicating that Ascll preferentially binds to bipolar regions. Overall, our analysis indicates a loss of neurogenic gene expression and motif accessibility during glial maturation that may prevent efficient reprogramming. 


\section{Introduction}

Neuron loss caused by disease and damage to the mammalian retina can lead to

permanent vision loss. While some species are readily capable of regenerating lost neurons,

mammalian retinas are not regenerative. In the mammalian retina, neuron loss caused by direct

damage to the retina leads to reactive gliosis of the Müller glia (MG), similar to that of astrocytes in the brain (Bringmann et al. 2009).

Teleost fish, by contrast, are capable of regenerating retinal neurons, including photoreceptors and ganglion cells, after damage. This regeneration is carried out by the MG, which respond to damage by generating progenitor-like cells, similar to those in the developing retina (Goldman 2014; Gemberling et al. 2013). This regeneration is accompanied by waves of gene expression and morphological changes to the $\mathrm{MG}$, regulated by epigenomic changes undergoes epigenomic changes after damage, but neurogenic programs are not re-expressed, and neuronal regeneration does not occur (VandenBosch and Reh 2019).

A critical difference between the fish $\mathrm{MG}$ and the mammalian MG in their response to

51 damage is in their expression of the proneural transcription factor Ascl1. In fish, Ascl1 is quickly

52 upregulated after damage, and is necessary for regeneration of new neurons (Ramachandran et

53 al. 2010; Fausett et al. 2008). In the murine retina, Ascl1 is expressed in retinal progenitors and

54 necessary for development of rods and bipolar cells (Ohsawa and Kageyama 2008); however it is

55 not expressed in mature MG and after damage or in disease models, mouse MG do not spontaneously upregulate Ascl1 (Bringmann et al. 2009; Ueki et al. 2015). We recently directed Ascl1 expression to mouse $\mathrm{MG}$ with a tetO transgenic approach to test whether Ascl1 expression 
days post-natal (P) stimulated MG to generate new bipolar neurons after NMDA damage (Ueki et al. 2015). In adult mice, however, Ascll over-expression in the MG is no longer sufficient to induce neurogenic potential, even in the presence of damage (Jorstad et al. 2017). In mature mice, the addition of the histone deacetylase trichostatin-A (TSA), in combination with Ascll overexpression and NMDA damage is required for neurogenesis; up to $30 \%$ of the Ascl1expressing MG produce functional bipolar- and amacrine-like interneurons, confirmed by single cell transcriptomics, electrophysiology, and electron microscopy (Jorstad et al. 2017). combination with HDAC inhibition suggests that epigenetic mechanisms may limit regeneration from the MG. In addition, even with the addition of HDAC inhibitors, the Ascl1-expressing MG only generate a subset of the neurons in the retina, suggesting that epigenetic factors may also limit the types of neurons that can be regenerated from mammalian MG. Thus, the expression of retinal progenitors. young MG (before eye-opening at P12), and adult MG. Analysis by ATAC and RNA sequencing demonstrates a clear trend in the loss of neurogenesis-related motif accessibility and expression.

To address the question of what distinguishes mature MG from late progenitors, we performed a transcriptomic and epigenomic comparison of FACS-isolated postnatal progenitors,

Ascl1, along with HDAC inhibition, does not fully recapitulate the multipotent progenitor state present in developing retina. Therefore, we asked whether changes in the transcriptome or epigenetic landscape might account for the difference in neurogenic potential between MG and Immature MG are found to have an intermediate epigenomic and transcriptomic profile. To test whether the intermediate profile of young MG regulates their neurogenic potential, we over- 
81 expressed Ascl1 in developing MG and have identified key restriction points in the neurogenic

82 potential of MG that correlate with changes in the accessible chromatin landscape.

83 


\section{Results}

\section{Chromatin Accessibility in Retinal Progenitors}

To determine the differences in the epigenomic landscape of retinal progenitors and developing MG, we used an Assay for Transposase-Accessible Chromatin (ATAC) sequencing to probe for differences in accessibility (Figure 1A). To isolate retinal progenitor cells, we used a knock-in Sox2-GFP mouse line that expresses GFP under control of the Sox2 promoter (Arnold et al. 2011). The great majority of Sox $2+$ cells at this age are retinal progenitors, though there is a small population of Sox $2+$ amacrine cells that can be distinguished from the progenitors by their high level of GFP. The retinas of P2 pups were dissociated into single cells and the GFP+ cells were sorted by Fluorescence-Activated Cell Sorting (FACS); the small number of strongly fluorescent amacrine cells were sorted separately from the more abundant progenitors (Figure S1). Sorted cells were used for two runs of ATAC-seq, reads were mapped and peaks were called using HOMER (Heinz et al. 2010) findPeaks. Two biological replicates were carried out (first run of $121 \mathrm{M}$ reads and $101 \mathrm{k}$ peaks, with a replicate with $95 \mathrm{M}$ reads and 68k peaks). When overlapped by BEDOPS (Neph et al. 2012), there were 40k common peaks between the samples, which were used for the subsequent analysis.

We previously characterized chromatin accessibility by DNase I in whole retinas at P0, P7 and Adult (Wilken et al. 2015). We compared the progenitor ATAC results with that from DNaseI-seq of the whole retina at the three ages. At P0, approximately $30 \%$ of the retinal cells are progenitors, while at $\mathrm{P} 7$ there are few progenitors remaining at the retinal periphery and none in the adult (Young 1985). Thus, we would anticipate the greatest overlap in accessible peaks between the progenitor ATAC-seq and the P0 retina. We found that progenitor-specific genes, such as Ascl1, show similar accessibility in the P0 retina and P2 FACS-purified progenitor cells 
108 (Figure 1B,C). Comparison of the progenitor ATAC-seq with older P7 and adult whole retina

109 DNaseI-seq showed a reduction in accessibility at regions near progenitor-specific genes,

110 consistent with the loss of progenitor cells as the retina matures.

111

112

Chromatin Landscapes in MG and Progenitors Have a High Level of Overlap

We compared the progenitor ATAC-seq data to our previously published mature adult

114 MG ATAC-seq to determine if there are specific molecular differences in chromatin accessibility

115 between these two cell types. The accessible chromatin in MG peaks was assessed in both FACS

116 purified MG from adult mouse retina, as well as from MG maintained in dissociated culture. In

117 previous studies, we found that a small amount of rod photoreceptor DNA and RNA is carried

118 along with the adult MG during FACS (Jorstad et al. 2017); however, when we maintain the MG

119 in dissociated culture, very few rods $(<0.1 \%)$ survive. Therefore, to reduce the contribution from

120 rod contamination in our downstream analyses, MG-specific regions of accessibility were chosen

121 that overlapped with DNase-seq from cultured P12 MG(Ueki et al. 2015). When we examine the

122 peaks that are present in both the freshly isolated MG ATAC-seq and the peaks from the cultured

$123 \mathrm{MG}$, we find they largely overlap (Figure S2).

When we then compared the MG accessible peaks with those of the progenitor cells, we

125 find that there is extensive overlap in overall accessibility. Approximately sixty percent of the

126 progenitor peaks (24,230 peaks) were shared between progenitors and MG, while P2 progenitors

127 had16k exclusive peaks, and the adult MG have 17k unique peaks (Figure 1F). Analysis of the

128 peaks that are unique to either progenitor cells or MG allowed some general conclusions: (1)

129 Both progenitor cells and MG have similar regions of accessible chromatin near genes that are 
131 and also had unique peaks near glial genes that were not present in the progenitor cells (Figure

132 1D). (2) Somewhat surprisingly, genes that are important for progenitor cell function (eg. Ascl1,

133 D111) had many of the same accessible regions near promoters in both the MG and the

134 progenitors (Figure 1E), though in many cases the P2 progenitors had more regions of

135 accessibility at these genes than the mature MG. The difference in accessibility between these

136 cell types varies from reduced peak height to a complete loss of some peaks. (3) Promoter

137 regions were over-represented in regions of shared accessibility, while peaks that were unique to

138 either MG or progenitor cells were predominantly found in intronic and intergenic regions

139 (Figure 1F;F'). Read density in these categories of accessibility showed that shared accessible

140 regions had an overall higher read density in broader regions, whereas cell type specific

141 accessible regions had narrow regions of read density (Figure 1F''). These differences suggest

142 that while promoter and major regulatory regions retain similar accessibility, many putative

143 regulatory regions differed in accessibility between these cell types, likely reflecting the

144 difference in their respective patterns of gene expression.

149 changing accessibility as regions that were either unique peaks called by Homer and overlapped 150 by BEDOPS (Neph et al. 2012) from P2 to Adult, or alternatively showed differences in tag

151 density by a $\operatorname{logFC}$ (fold change) $>2$ in the top 1000 regions of Differential Accessibility (DA).

152 These two different pipelines gave similar results for gene ontology and binding motif annotation

153 (Tables S1, S2). 
When we carried out gene ontology (GO) analysis for those regions that had greater

155

156

157

158 accessibility in the progenitor cells than in the MG (Loss of Accessibility or LOA), we found these regions were associated with genes that were enriched for GO terms of Neural Development/Neurogenesis and Developmental Process/Cell Differentiation (Figure 2A). By contrast, the peaks that were not accessible in retinal progenitors, but present in mature MG (ie. Gain of Accessibility or GOA), were associated with genes that were enriched for GO terms of more general cell function: e.g. metabolic and cell process genes (Figure 2B). Those accessible regions present in both cell types are also enriched primarily in metabolic genes (Table S1). We next performed ATAC-seq on FACS-purified immature P8 MG to better understand the dynamic changes in the accessible chromatin that occur as MG mature (Figure S3). For the P8 sample, there were 49.6M reads and 16k peaks by Homer findPeaks, of which $98.35 \%$ overlapped with the P7 DNaseI whole retina dataset. We carried out DA analysis between the P8 and adult MG, and the progenitor cells and P8 MG. Interestingly, we found GO terms of Nervous System Development and Generation of Neurons as some of the top Biological Process terms in the P8 MG accessible regions that are lost or reduced in the mature MG cells (Figure 2C). Thus, it appears that many of the putative cis-regulatory regions near genes associated with neurogenesis are still accessible in the immature P8 MG, and suggests these cells may be more amenable to reprogramming than mature MG.

The peaks of accessible chromatin near genes associated with neurogenesis that are unique to progenitor cells (ie. not present or reduced in the mature $\mathrm{MG}$ ) may be relevant to the differences between these cells in their ability to generate neurons. We analyzed these putative neurogenesis-related cis-regulatory elements for transcription factor binding motifs using Homer findMotifsGenome against randomly selected background regions. We found that the top five 
177 transcription factor motifs present in the peaks unique to progenitor cells (LOA) were - CTCF, 178 Lhx (eg. Lhx2), bHLH (eg. NeuroD1), HMG (eg. Sox2) and nuclear receptor (Figure 2D).

179 Progenitor cells are known to express several members of each of these transcription factor 180 families; for example, the proneural bHLH factors Neurog2, Olig 2 and Ascl1 are all expressed 181 in retinal progenitors at this stage (Brzezinski et al. 2011). When comparing Eboxes with 182 specificity to Ascl1 and Neurog2, we found that Ascl1-specific Eboxes had far more distinct 183 central enrichment, especially in regions of progenitor-specific accessibility, whereas Neurog2 184 Eboxes are centrally depleted (Figure S4A,B). Factors such as Lhx and Sox are known to be 185 expressed in both progenitors and $\mathrm{MG}$, and thus their presence in progenitor-specific accessible domains is likely indicative of restructuring of regulatory regions during development. While the accessible chromatin regions specific to progenitors were enriched for proneural transcription factors, the accessible regions present in the $\mathrm{MG}$, but not present in the progenitors (GOA), have a very different set of enriched transcription factor binding motifs. Although CTCF and Lhx motifs were still among the top 5, the NFI (eg. NFIx) binding motif is enriched in those accessible regions unique to the MG (Figure 2E). This motif was also present in the accessible regions that differ between the immature P8 MG and the progenitors, making this an early marker of the unique MG epigenetic landscape (Figure 2F). The motifs enriched in 194 the MG-specific accessible chromatin regions may well reflect the importance of Lhx and NFI transcription factors in MG maturation(Hägglund et al. 2011; de Melo et al. 2016; Gordon et al. 2013; Shu et al. 2003) Central enrichment modeling also highlighted the differences between progenitors and MG. For example, progenitor-specific accessible regions were centrally enriched for predicted 199 Ascl1 motifs, with much lower accessibility in the adult MG at these sites (Figure 2G). The 
opposite is true for the NFIx motif: adult MG-specific accessibility profile showed much higher

214 (Ascl1) or glial (Nfix) cell fate.

\section{Immature MG Expression is Intermediate to Progenitors and Mature MG}

To better understand the molecular basis for the difference in accessibility between the and mature MG (Figure 3A). MG were sorted using Rlbp-creER:flox-stop-tdTomato reporter 
differentially expressed in MG (Gain of Expression, GOE) or in progenitors (Loss of Expression, LOE) by $\log C P M$ (counts per million) values (Figure 3B). Genes were clustered via k-means clustering, and GO terms were associated with specific clusters (Figure 3D, Supplementary Tables 4, 5). Genes that showed marked increases over the period from P2 to adult MG had Biological Process terms of Response to Stimulus, and Biological Regulation, while genes that were highly expressed in progenitors, but expressed at much lower levels in MG were associated with GO terms that included Cell Cycle, Nervous System Development and Neurogenesis

230 (Figure 3D, Supplementary Table 5). Thus, the changes in gene expression between progenitors and MG are very similar to the changes in DNA accessibility. immature MG expressed many of the genes that are expressed in the retinal progenitors, albeit at 234 a lower level than the progenitors, but in addition they expressed genes more characteristic of 235 mature MG (Figure 3D). The heatmap shows that four of the five clusters of "MG-specific" 236 genes had the greatest increase between P2 and P8. Genes that lost expression most rapidly, in 237 the tan cluster are primarily associated with the cell cycle. In contrast, the cluster (blue) most 238 closely associated with the GO terms of Neurogenesis and Nervous System Development 239 declined over the first postnatal week more gradually, but had very low levels of expression in mature MG (Figure 3D). Comparing expression up and down across MG maturation revealed an

241 intermediate level of expression in young MG with progenitor gene expression retained at 242 relatively high levels through $\mathrm{P} 8$.

Are the changes in gene expression between MG and retinal progenitors reflected in their 244 chromatin accessibility? To answer this question, we compared the genes that change in their expression (GOE, LOE) with the developmental ATAC categories (GOA, LOA). We directly 
compared the GOE and LOE genes to accessibility categories (GOA, LOA, SA [Shared Accessibility]) using gene annotations by GREAT to neighboring genes, yielding a percent of total genes for GOE and LOE categories for each accessibility profile (Figure 3C, Supplementary Table 6). The best correlation between changes in gene expression and changes in accessibility was found in the adult MG: GOA regions are primarily associated with genes that increased in expression (Figure 3C, right hand bars). This correlation was not as clear in the other categories. Accessible chromatin regions that were shared between progenitors and MG were associated with genes that showed a small reduction in expression between progenitors and MG. However, accessible chromatin regions that were specific to progenitors were not necessarily associated with genes that were more highly expressed in progenitors than MG. Despite the lack of good correlation between accessibility and gene expression on a global level, analysis of specific genes and sets of genes are informative. For example, the regions of lost expression and accessibility (LOE/LOA) are best associated with Neurogenic GO categories, and this trend is continued in comparisons from P8 to Adult MG LOE/LOA (Supplementary Table 7).

By overlapping the ATAC-seq data with the gene expression results, we were able to better define some of the differences in transcription factor expression and putative binding at gene-associated regions that might be regulating the difference in neurogenic competence between these two cell types. For example, LOE/LOA regions were enriched for binding motifs for proneural bHLH factors, whereas those genes that gain accessibility and expression (GOE/GOA) were enriched for binding motifs for NFI and ROR (Figure 3E). These results are in line with those obtained from the analysis of the ATAC seq results (described above), indicating that the binding motifs that differed between these cells were associated with and may regulate those genes that similarly changed in expression. Transcription factors that bind to these motifs 
were also found to change expression in accordance with changes in motif accessibility (Figure 3F,G). Interestingly, those putative cis-regulatory elements that were accessible in both MG and

271 progenitors, but that lost expression in mature MG (LOE/SA) are associated primarily with genes

272 for cell cycle. These regions were enriched for the binding motif for the cell-cycle regulator E2F

273 transcription factor (Figure 3D,E). This suggests that the differences between progenitor cells

274 and MG in their cell proliferation are unlikely to be regulated by changes in chromatin

275 accessibility.

276

Ascll is Sufficient to Induce Neurogenesis in Immature MG

Our previous results showed that histone modifications may play a role in limiting the competence of MG to regenerate neurons: HDAC inhibition is necessary for Ascl1 to reprogram adult MG to neurogenic progenitors (Jorstad et al. 2017). Given the increased chromatin accessibility at progenitor genes of immature $\mathrm{MG}$ that we found at $\mathrm{P} 8$, we predicted that it might be possible to reprogram glia to a neuronal fate at P8. To test the hypothesis, we overexpressed Ascl1 in retinal progenitors and $\mathrm{MG}$ at various times during postnatal development. In order to trace the lineages of retinal progenitors and $\mathrm{MG}$, we used a tamoxifen-inducible creER mouse driven by one of two promoters to activate a fluorescent reporter. For control mice, we used

286 Glast-CreER:flox-stop-CC-GFP; mice received an intraperitoneal injection of tamoxifen to 287 initiate the recombination at $\mathrm{P} 0, \mathrm{P} 4, \mathrm{P} 8$, or $\mathrm{P} 12$. The promoters are glial-specific in adult mice, 288 but are expressed in retinal progenitors at P0. When cells were lineage traced at P0, the late-born 289 types of neurons are present in the progeny: 72.6\% of GFP+ cells had a photoreceptor fate, $29022.7 \%$ were bipolar cells, and the remaining cells were MG (Figure 4A,C). These are similar to 291 the ratios found when these cells that are birth-dated at this age in mice (Young 1985). By P4, 
292 the percentage of GFP+ neurons was significantly reduced with only $2.72 \%$ of cells having a

293 bipolar cell fate, while the remainder throughout the retina were MG. At the far retinal periphery

294 however, some retinal progenitor cells were still present at P4 and so some rods and bipolar cells

295 were observed in this area (Figure S4). In mice older than P4, 100\% of GFP+ cells were MG.

296 From P8 and on, 100\% of GFP+ cells were MG. These results are consistent with previous

297 reports that neurogenesis in the retina ends after P4, with the exception of some neurogenesis at

298 the periphery.

To determine whether immature MG can be reprogrammed to a neurogenic state with

300

301

302

303

304

305

306

307

308

309

310

Ascl1 alone, we drove expression of Ascll at the same ages that we had used to trace WT cells.

We used a previously described system for driving Ascll in MG: Glast-CreER;Flox-stop-LNL-

tTA;TetOCMV-Ascl1-ires-GFP (Ueki et al. 2015; Jorstad et al. 2017). With the induction of

Ascl1 expression at $\mathrm{P} 0$, ratios of GFP+ neurons were not significantly different from WT

lineages. This suggests that the level of Ascl1 is expressed at sufficient levels in retinal

progenitors to sustain neurogenesis, and that additional Ascl1 has little effect. At P4 and P8, however, over-expression of Ascl1 in immature MG has a dramatic effect on the cells: $75 \%$ and $65 \%$ of GFP + cells, respectively, are now bipolar neurons, with few-if any-photoreceptor neurons. When we delay induction of Ascl1 to P12, however, 100\% of GFP+ cells are now MG, and at this age, expression of Ascll alone is no longer sufficient to reprogram the MG to neurons, similar to results previously reported(Ueki et al. 2015; Figure 4A,D). These results show there is a rapid change in the competence of cells to generate rods with Ascl1overexpression summarized in Figure 4B. Some major change (Transition [T]1) occurs at P4 in the progenitor cells as they become MG to restrict the ability of Ascll to generate rods. A second major change (Transition [T]2) appears to control the ability of MG to generate bipolar cells, and 
this occurs after P8. After this time, damage to the retina and inhibition of histone deacetylases is needed to induce neurogenesis from the $\mathrm{MG}$.

\section{Retinal Neuron Chromatin Overlaps Similarly with Progenitor and MG}

The changes in neurogenic competence that occur as cells transition from progenitor cells to $\mathrm{MG}$ even with forced Ascl1 expression might be due to changes in their epigenome. To determine if DNA accessibility might underlie these differences in (1) neurogenic competence, and (2) fate restriction to predominantly bipolar cell neurogenesis, we compared ATAC-seq data from progenitor cells, MG, rod photoreceptors (Hughes et al. 2017), and bipolar cells (Jorstad et al. 2017) (Figure 5A). We found that both progenitor cells and adult MG accessible regions overlapped with rods by approximately $19 \mathrm{k}$ peaks or $47.5 \%$ of glial/progenitor-accessible domains (Figure 5B,D). A similar analysis of bipolar cells and retinal progenitors or MG gave very similar results: bipolar cell accessibility overlapped with progenitor accessible regions by approximately $20 \mathrm{k}$ peaks (50\% of progenitor domains), and with MG accessible regions by approximately $17 \mathrm{k}$ peaks (42.7\% of glial domains) (Figure S6). For all overlaps, GO categories were similar between comparisons to progenitors or MG (Table S8). As Otx2 is involved downstream of Ascl1 in the generation of both cell types (Omori et al. 2011), we looked for central enrichment of this motif in both neuronal-specific and MG-shared accessible regions. For rods, the rod-specific accessible regions (ie. not present in the MG or the progenitor cells) are strongly centrally-enriched for Otx2 binding sites (Figure 5C,E, S6). We did not see a similar central enrichment for Otx 2 in the accessible regions shared between rods and progenitor cells or rods and MG. A similar analysis of the overlapping and uniquely accessible regions for bipolar cells and either MG or retinal progenitors gave strikingly similar results: the accessible regions 
that are unique to bipolar cells and not shared with progenitor cells or MG show strong central specific regions - when comparing to either MG or progenitors - were enriched for homeobox,

344 and CTCF domains, though in comparison to progenitors, rod photoreceptor cells may have an

345 additional enrichment for bHLH binding motifs (Figure 5F, Table S2). Shared accessible regions in both comparisons showed enrichment for CTCF, Klf, and ETS binding motifs. Bipolar cell accessible regions that were shared with both progenitor cells and MG also showed enrichment

348 for these TF binding motifs. Interestingly, in both comparisons, bipolar cell-specific accessibility

349 demonstrated enrichment not only for homeobox domains, but also for proneural bHLH domains

350 (Figure 5G). The presence of bHLH accessibility in bipolar cells may underlie the fact that MG 351 primarily generate bipolar cells with the overexpression of Ascll. 
Because the role of Ascll appears to be a key difference between bipolar cell and rod photoreceptor cells, we took a closer look at its binding in accessible and inaccessible domains.

363 Casey et al 2018 recently demonstrated that ES cell reprogramming with Ascl1 revealed a

364 particular class of accessible regions with repeated Ebox motifs (Casey et al. 2018). Similar to

365 that study, we find that those regions of the DNA that are inaccessible in MG or progenitors, but

366 bind Ascl1 when over-expressed (pioneered sites) had on average 4.7 E-boxes per peak, while

367 other Ascl1-bound sites in MG have fewer than 2 E-boxes on average (Figure S7). We

368 additionally assayed E-box number in Rod and Bipolar accessible domains, but found no

369 difference in average number of E-boxes per peak (DNS).

In addition to this predictive analysis, we compared accessibility domains using Ascl1

371 ChIP-seq from both P0 retinas as well as from Ascll overexpression in cultured MG (Figure

$3725 \mathrm{H})$. While developmental Ascll largely binds in shared and progenitor accessibility domains as

373 well as some non-glial sites, ectopic Ascl1 has a strong distinct niche in Adult MG and in so-

374 called "radical" binding sites (Figure S7). In observation of regions predicted to be enriched in

375 Ascl1 binding in retinal neurons, we found that Ascll also binds more strongly to bipolar

376 accessible domains as compared to rod accessible domains (Figure 5I). Tag density was highest

377 for progenitor and glial-shared accessible regions, but there was distinct central enrichment for

378 Ascl1 binding in bipolar-specific accessible domains (Figure 5J,K). Binding of Ascl1 ectopically

379 and Ebox patterning in novel accessible domains demonstrate that expression of this TF in MG

380 may further direct fate decisions more towards bipolar interneurons than photoreceptor neurons. 


\section{Discussion}

In this study, we have explored the epigenetic profile of retinal progenitors and MG alongside their gene expression profile as these cells change in their neurogenic potential. We have demonstrated epigenetic evidence of putative cis-regulatory elements that change in accessibility through development and potentially regulate the maturation and neurogenic

387 allows for improved reprogramming to bipolar neurons, thus revealing transition states in the neurogenic potential of immature mammalian glia.

In order to explore the potential mechanisms for regulation of epigenetic regenerative capacity in the mammalian MG, we performed ATAC-seq on postnatal neurogenic retinal progenitors as well as on developing and mature MG. We found that the epigenomic landscape of progenitors and mature MG were very similar, with approximately $60 \%$ of progenitor accessible domains shared with mature $\mathrm{MG}$, consistent with similarities in expression between 394 progenitors and MG (Jadhav et al. 2009; Nelson et al. 2011). These shared regions include glial395 expressed genes as well as progenitor-specific genes and were particularly enriched around 396 promoter regions. Cell-type specific accessible regions were enriched in intronic and intergenic 397 regions of the genome, consistent with previous evidence that enhancers may be more dynamic 398 during development(Aldiri et al. 2017).

Accessible regions that were enriched in the retinal progenitors were specifically

400 associated with developmental and neurogenic genes. This was reinforced by expression 401 analysis, demonstrating that progenitor-specific genes, enriched in GO categories associated with 402 early development and neurogenesis, showed more than $2 \operatorname{logFC}$ compared with MG. Many regions of accessibility that were reduced or lost as the MG mature were associated with these 
404

405

406

407

408

409

410

411

412

413

414

415

416

same genes that lost expression during postnatal development, reinforcing that accessibility correlates with expression. Motif enrichment analysis of the progenitor-specific accessible domains revealed specific enrichment in bHLH binding domains. This class of transcription factor is well-characterized as part of the retinal developmental process, though there are many variations of E-box motifs in the genome that are relatively specific to different bHLH domains. Aydin et. al 2019 recently described variations of E-box specificity that contribute to neuronal subtype (CAGSTG for Ascl1 and CAKATG for Neurog2) (Aydin et al. 2019); we found a nonspecific Ebox enriched by HOMER, consistent with progenitor cell potential to generate multiple types of neurons (both excitatory and inhibitory), with the fourth position in the motif being equally probable to be A or C. However, specific assaying for specific Ebox motifs demonstrates a preference for the Ascl1 motif throughout. The loss seen in bHLH binding motifs in the transition from progenitors to MG confirms that these transcription factors reflects the decline in expression of Ascll and related bHLH TFs as progenitors transition to MG. As MG mature, the cells increased in both accessibility and expression associated with genes important for glial function. There was a correlation between increases in nearby accessible chromatin and genes that increased in expression during glial maturation. Young MG in particular had a unique intermediate profile. Glial genes that were highly increased in expression in adult MG gain accessibility before P8, but this is not accompanied by a similar drop in progenitor gene expression.

Cis-regulatory accessibility continued to change and develop during MG maturation.

While many enriched motif classes were common between decreasing and increasing accessibility, the NFI binding motif was uniquely enriched in the developing glia. NFI domains were similarly enriched surrounding genes that increase expression and gain accessibility, 
427 indicating a putative role in the development and maturation of the glial fate. These NFI domains

428 were enriched in accessible regions even in the younger P8 MG, consistent with evidence for an

429 intermediate profile of immature MG. NFI transcription factors are relevant to the developing

430 glia as they are well known for their role in CNS glial development (Shu et al. 2003; Heng et al.

431 2015; Deneen et al. 2006). In addition, NFIa/b/x are expressed in retinal progenitors and MG,

432 and conditional knockouts of these transcription factors in the developing retina are associated

433 with defects in gliogenesis and the production of bipolar neurons (Clark et al. 2019). As

434 progenitors develop, there is an acquired association of NFI motifs with Lhx2 binding sites,

435 which may be related to guiding the neurogenic potency of late retinal progenitors (Zibetti et al.

436 2019).

Though RNA-seq and ATAC data demonstrate epigenomic changes that co-occured with changes in gene expression, not all gene expression changes were associated with changes in accessibility. This is best demonstrated with cell cycle and proliferation-related genes. These regions are associated with E2F binding motifs, consistent with their roles in regulating mitotic

441 proliferation (Wu et al. 2001; Stevaux and Dyson 2002). Expression of genes associated with the 442 mitotic cell cycle declined as progenitors withdrew from the cell cycle. However, regions of 443 accessibility associated with these genes changed little in their accessibility between progenitors 444 and MG. This suggests that the loss of proliferative capacity in maturing MG may not be limited 445 by epigenetic accessibility.

447 the progenitors and adult MG. Since efficient reprogramming of mature MG requires a

448 combination of Ascl1 and HDAC inhibition, we postulated that the P8 MG might be more 449 efficiently reprogrammed to a neuronal fate with Ascll alone. We found that induction of Ascl1 
alone in immature MG was indeed capable of inducing a neuronal/bipolar cell fate, consistent with our hypothesis that epigenomic changes in accessibility limit mature MG from regeneration. Interestingly, the P8 MG already appeared to be fate restricted with respect to the types of neurons produced from Ascl1 over-expression, with two distinct transition periods. In newborn mice, lineage tracing and birthdating studies have shown that progenitors generate three types of neurons: rods, bipolar cells and amacrine cells (Young 1985); however, early in MG development (eg. P4), the MG cells lose their ability to generate photoreceptors and amacrine

457 cells (T1), even with Ascl1 over-expression. Somewhat later in MG development (eg. P10) as 458 the cells mature, they lose their ability to generate bipolar cells from Ascl1 over-expression 459 alone, though the addition of HDAC inhibitors and injury can restore their neurogenic potential 460 (Ueki et al. 2015; Jorstad et al. 2017).

Do changes in accessible chromatin account for the bipolar fate restriction that occurs

462 during the T1 transition? To address this question, we compared the open chromatin landscape of 463 bipolar cells and rod photoreceptors to determine the degree of similarity between these neuronal 464 cell types and MG or progenitors. Overall, the shared accessible regions between progenitors and 465 either type of neuron (rod or bipolar cell) were very similar to the shared regions between MG 466 and these types of neurons. Moreover, the newly accessible sites in rods or bipolar cells were not 467 present in either progenitors or MG. Thus, the degree of similarity in accessible chromatin 468 between progenitors or MG, on the one hand, and rods or bipolar cells, on the other, are not 469 sufficient to explain the bias in bipolar cell generation from MG. Nevertheless, in comparing 470 neuron-specific accessible regions from rods and bipolar cells, we found that bipolar cell-specific

471 accessible regions were more highly enriched for bHLH motifs than rod-specific accessible regions. Also, over-expression of Ascl1 in MG results in Ascl1 binding to bipolar cell-specific 
473 accessible regions over rod-specific regions. Similar enrichment for Ebox accessibility in a

474 variety of bipolar cells was also shown by Murphy et al (Murphy et al. 2019), and this may help

475 to explain why the overexpression of Ascl1 in MG preferentially generates bipolar cells in vivo,

476 though the factor is necessary for the generation of both bipolar cells and photoreceptors during

477 development.

In sum, we have characterized the ways in which the accessible chromatin landscape

479 changes as retinal progenitors differentiate into MG. Developing MG lose expression of

480 neurogenic genes and accessibility of related cis-regulatory elements, and gain accessibility of

481 glial-defining NFI binding sites. However, young MG demonstrate intermediate profiles. By P8,

482 MG demonstrate early gains in NFI binding sites, while retaining progenitor-like expression and

483 accessibility. This intermediate profile translates into neurogenic potential: prior to P12

484 overexpression of Ascl1 alone is sufficient to induce neurogenesis in MG. The affinity for

485 bipolar neurons appears to be in part due to the preference of Ascll for binding to bipolar cell

486 specific accessible regions, and not due to inherent overlaps in MG chromatin accessibility with

487 bipolar cells vs rods. Overall, our results show unique transition states in the development of

488 glial cells that restrict their neurogenic potential, which correlate with changes in the epigenome.

490 Methods

$491 \quad$ Mice

492 All mice were housed at the University of Washington and treated with protocols approved by

493 the University of Washington's Institutional Animal Care and Use Committee. Mice expressed

494 Cre-recombinase under the Glast promoter from Jackson Labs with Rosa-flox-stop-tTA (Jackson

495 labs) in combination with either tetO-mAscl1-ires-GFP (M. Nakafuku University of Cincinnati) 
or CCGFP. Mice having EGFP knocked-into the Sox2 open reading frame were obtained from Jackson Laboratories (Stock: 017592) and bred to generate P2 litters(Arnold et al. 2011). Rlbp1CreERT2 mice were crossed to R26-stop-flox-CAG-tdTomato mice (Jackson Labs, also known as Ai14; 129SvJ background). Mice of both sexes were used for this study and analyzed together in their respective treatment groups.

\section{Injections}

Intraperitoneal injections of $50 \mu \mathrm{l}$ of tamoxifen (Sigma) at $100 \mathrm{mg} / \mathrm{kg}$ in corn oil were given to induce expression of Ascl1 and GFP. Tamoxifen was administered once for P0 or P4 induction, for 2 consecutive days in mice aged P6-P9, or 4 consecutive days for older ages.

\section{IHC and cell counts}

For lineage tracing with GFP and Ascll induction, animals were euthanized and the eyes removed for dissection and removal of the cornea and lens. Eyes were then fixed in $4 \%$ PFA in PBS for $1 \mathrm{~h}$ before being transferred to a 30\% sucrose in PBS solution and kept overnight at $4{ }^{\circ} \mathrm{C}$. Eyes were then frozen at $-80{ }^{\circ} \mathrm{C}$ in O.C.T. (Sakura Finetek) and sectioned to $14 \mu \mathrm{m}$ by cryostat (Leica). Slides were incubated at room temperature in blocking solution (10\% normal horse serum, $0.5 \%$ Triton $\mathrm{X}-100$, in PBS) for $1 \mathrm{~h}$. Slides were then incubated overnight at $4{ }^{\circ} \mathrm{C}$ in primary antibody diluted in blocking solution. Slides were then washed three times in PBS for 15 minutes each before a $1 \mathrm{~h}$ room temperature incubation in secondary antibodies (Life Technologies) in PBS. Slides were washed once before being incubated 5-10 minutes with 1:10,000 DAPI (Sigma) in the dark. At this point, slides were washed three times in PBS and coverslipped with Fluoromount-G (SouthernBiotech). Primary antibodies: goat anti-Sox2 (Sanda 
Cruz, 1:500), mouse anti-HuC/D (Invitrogen, 1:100), chicken anti-GFP (Abcam, 1:500), goat anti-Otx2 (R\&D Systems, 1:100), rabbit anti-Recoverin (Millipore, 1:1000).

Section imaging was performed using an Olympus Fluoview confocal microscope, and random fields throughout the retina were sampled for cell counts. Cell types were identified and counted by localization within the retina, cell morphology, and marker co-staining. Significance

524 values between treatments were determined by one-way ANOVA with a post-hoc tukey test or by t-test.

\section{FACS}

528 FACS protocol according to (Wohl and Reh 2016)

529 Retinas were isolated via dissection away from surrounding tissues and then washed in PBS.

530 Fluorescence was confirmed via live imaging under an inverted fluorescent microscope (Zeiss).

531 Retinas were then incubated on a nutator in Papain and DNase I for 10 min at $37{ }^{\circ} \mathrm{C}$. Retinas

532 were then triturated to generate a single-cell suspension and transferred to a tube containing an

533 equal volume of Ovomucoid. The suspension was then spun down at $300 \mathrm{~g}$ for $10 \mathrm{~min}$ at $4{ }^{\circ} \mathrm{C}$.

534 Cells were resuspended in a solution of 100:1:1 Neurobasal:DNase:Ovomucoid, and passed

535 through a $35 \mu \mathrm{m}$ filter before sorting using a BD FACSAria III Cell Sorter (BD Biosciences).

536 Gating was performed to isolate intact cells from debris and to isolate positive fluorescent glial 537 or progenitor cells. Progenitor cells were isolated from Sox $2+$ Amacrine cells by removing the 538 higher fluorescent neuronal population. Positive fractions containing fluorescently labelled MG 539 were then spun down at $300 \mathrm{~g}$ and resuspended for the appropriate assay. 
542 Purified cells from live-cell FACS were input into a $15 \mu l$ transposase reaction with an input of

543 100k cells in a protocol modified from the Greenleaf lab (Buenrostro et al. 2015). Transposition

544 was carried out with reagents from the Nextera DNA Sample prep kit: $7.5 \mu 1$ XX TD Buffer, 0.75

$545 \mu 1$ Tn5 Transposases, and nuclease-free water to $15 \mu 1$. The reaction was mixed and incubated at

$54637^{\circ} \mathrm{C}$ for one hour before being purified with the Qiagen Reaction Cleanup Minelute kit and

547 eluted into $10 \mu \mathrm{l}$. Libraries were prepared through subsequent PCR using Illumina Nextera kit

548 (Cat. No. FC-121-1030) using a test qPCR output to estimate the number of cycles necessary to

549 properly amplify the library. Amplified libraries were purified with the Qiagen PCR cleanup

550 minelute kit and eluted into $20 \mu \mathrm{l}$. Library QC was performed using gel electrophoresis, and

551 quantitated on a Qubit 3.0 Fluorometer with the dsDNA HS Assay kit and A260/280 and

552 A260/230 checked by nanodrop before sending for Illumina NextGen sequencing on a Next Seq

553500 in rapid mode employing a paired-end 50 base read length sequencing strategy (Seattle

554 Genomics). Adapter and barcode sequence were removed from the reads and low-quality

555 sequences (Phred score <33) were removed using Trim Galore (Krueger 2015). Remaining reads

556 were mapped using Bowtie2 (Langmead and Salzberg 2012), marking duplicate reads with

557 Picard (http://broadinstitute.github.io/picard/), and removing reads using Samtools (Li et al.

558 2009). Alignment data was normalized for coverage using a custom R script

559 (https://rpubs.com/achitsaz/98857) and visualized using the Integrated Genomics

$560 \quad$ Viewer(Robinson et al. 2011)

561

562

Peak calling and comparison

563 Peaks were called using HOMER (Heinz et al. 2010) findPeaks dnase style with a minimum

564 distance of 415 and size of 150 . Bedops -e 1 and $-\mathrm{n} 1$ functions were used to compare peak files 
565 for binary peak differences (Neph et al. 2012). For differential accessibility comparisons, the R

566

567

568

569

570

571

572

573

574

575

576

577

578

579

580

581

582

583

584

585

586

587

package EdgeR (Robinson et al. 2010) was used to compare all peak regions between two ATAC

samples against the reads of each file as previously described. The RSubread (Liao et al.

2019)function featureCounts was used to generate a matrix of counts per million across all

peaks. The counts matrix was filtered against low-reads rows and in the case of any sample that

is more deeply sequenced than another, the EdgeR (Robinson et al. 2010) function thincounts

was used to thin one sample randomly to the level of the lower depth of sequencing. Dispersion,

fitting and differential signal testing were performed using negative binomial generalized linear

models as specified in the edgeR guide. Cumulative differences in accessibility at each gene

were calculated as the sum of the fold differences of all peaks nearest to each gene. Peaks of

interest were identified by selecting for those with a $\log 2 \mathrm{FC}$ above 2 , and the top $1 \mathrm{k}$ peaks up

and down were selected by $\log 2 \mathrm{CPM}$. The peakIDs for each of these regions were used to

generate new peak files and perform further analysis.

\section{Ascl1 Chromatin Immunoprecipitation-Sequencing (ChIP-Seq):}

P0 retinas or cultured, post-natal day 12, Müller glia (+/- Ascl1 overexpression, rtTA

germline:tetO-Ascl1-ires-GFP mice \pm doxycycline) were digested with papain/DNase to single

cells and fixed with $0.75 \%$ formaldehyde for 10 minutes at room temperature. Sonication was

performed with a probe sonicator (Fisher Scientific): 12 pulses, $100 \mathrm{~J} /$ pulse, Amplitude: 45, 45

seconds cooling at $4{ }^{\circ} \mathrm{C}$ between pulses. Immunoprecipitation performed with $40 \mu \mathrm{L}$ anti-mouse

IgG magnetic beads (Invitrogen Cat: 110.31) and $4 \mu \mathrm{g}$ mouse anti-MASH1 antibody (BD

Pharmingen Cat: 556604) or $4 \mu \mathrm{g}$ mouse IgG against chromatin from 5 million cells per

condition according to Diagenode LowCell Number Kit using IP and Wash buffers as described 
in (Castro et al. 2011). Libraries were prepared with standard Illumina adaptors and sequenced to an approximate depth of 36 million reads each. Sequence reads (36 bp) were mapped to the mouse mm9 genome using bwa (v 0.7.12-r1039). Merging and sorting of sequencing reads from

591 different lanes was performed with SAMtools (v1.2). The HOMER software suite was used to

592 determine and score peak calls ('findPeaks' function, v4.7) as well as motif enrichment

593 ('findMotifs' function, v4.7, using repeat mask). For STATi and control Ascl1 ChIP-seq, reads

594 were aligned to the mm10 genome using Bowtie2. The .sam files were converted to sorted .bam

595 files using SAMtools. MACS2 was used to call peaks with default settings using the broad peaks

596 annotation. Peak overlap analyses were performed using Bedops. The control Ascl1 ChIP-

597 seq .bam file was downsampled by a factor of 0.69 to normalize the number of mapped reads

598 over the common peaks found between treatment and control samples. This downsampled .bam

599 file was used for all analyses. Differential accessibility analysis in Ascll ChIP-seq peaks was

600 determined using edgeR as detailed in the edgeR user guide.

601

602

603

Bulk RNA seq

604 For RNA-Seq, FAC-sorted cells were resuspended into Qiazol and RNA was extracted using the

605 Qiagen RNeasy MinElute kit. Samples were tested for QC using nanodrop, and sent for

606 sequencing. $500 \mathrm{ng}$ per sample $\left(50 \mathrm{ng} \mathrm{H}^{-1}\right)$ was sequenced on an Illumina HiSeq and reads that

607 passed Illumina's base call quality filter were mapped to mm10 using TopHat v2.0.12. To

608 generate counts for each gene using htSeq-count v0.6.1p1, in "intersection-strict" overlap mode,

609 genes with zero counts across all samples were removed, and data normalized using edgeR

610 v3.12.0. Further analysis was done using Bioconductor and R (version 3.2.3). 
612 EdgeR was used to compare reads across RNA-seq samples according to the published manual

613 guide for exactTest(Robinson et al. 2010). Prior to testing, samples with higher reads were

614 thinned to the level of other samples using thinCounts. Dispersion, fitting and differential signal

615 testing were performed using negative binomial generalized linear models as specified in the

616 edgeR guide. Differential expression was filtered to generate the top $1 \mathrm{k}$ genes up and down

617 across development by selecting for genes with a $\log \mathrm{FC}$ greater than two, and top genes of this

618 category were selected by highest $\log 2 \mathrm{CPM}$. The top genes were clustered using hclust to

619 perform ward D2 agglomeration with Euclidian distances. Genes were filtered against annotated

620 ATAC peaks by dplyr.

621

622 Cell culture and expression

623 From (Pollak et al. 2013)

624 MG from postnatal day (P)12 mice were cultured [Neurobasal + N2, epidermal growth factor

625 (EGF), 10\% fetal bovine serum (FBS)] as previously described with $1 \mu \mathrm{M}$ 5-ethynyl-2'-

626 deoxyuridine (EdU). Lentiviral particles were added in Optimem (Gibco) or neural medium

627 (Neurobasal + N2, B27, 1\% tetracycline (tet)-free FBS), and 3-6 hours later medium was

628 replaced. hBDNF (R\&D Systems, $10 \mathrm{ng} / \mathrm{ml}$ ), bFGF (R\&D Systems, $100 \mathrm{ng} / \mathrm{ml}$ ) and rGDNF

629 (R\&D Systems, $10 \mathrm{ng} / \mathrm{ml}$ ) were added for longer cultures. 4-Hydroxytamoxifen (4-OHT; Sigma)

630 was included where indicated at $10 \mu \mathrm{M}$.

631

632

633

Homer annotatePeaks.pl 
634 For basic annotation of ATAC peaks, the Homer function annotatePeaks.pl was used (Heinz et

635 al. 2010). While in most cases, peak annotation to genes was accomplished with the online tool

636 GREAT (McLean et al. 2010), annotating each region for closest gene, the basic usage was used:

637 annotatePeaks.pl < peak file $>$ mm9 $>$ output.txt

638 For stats on genome location:

639 annotatePeaks.pl $<$ peak file $>$ mm9 -annStats $>$ output_stats.txt

640 For annotation of specific motifs, a position weight matrix was either taken from the homer

641 database, from the findmotifs function, or was generated using seq2profile.pl in Homer, and was

642 used in annotatePeaks as such:

643 annotatePeaks.pl < peak file $>$ mm9 -m pwm.motif $>$ output_motif.txt

644 However, for the generation of lineplots, a table of $10 \mathrm{bp}$ bins for motif enrichment were

645 generated for graphing with ggplot2:

646 annotatePeaks.pl < peak file> mm9 -m pwm.motif -size 2000 -hist $10>$ output_plot.txt

647 For motif co-occurrence:

648 annotatepeaks.pl <peak file> mm9 -size 2000 -hist 20 -m <motifs of interest> -cpu 10 -matrix

649 fileout $>$ fileout.motif.freq

650

651

652

Homer Motif discovery

653 To discover enrichment of predicted DNA binding motifs for further analysis, we employed the

654 Homer function findMotifsGenome.pl using suggested basic usage settings. From there, we

655 identified top motifs from the homerResults output

656 
657

658

659

660

661

662

663

664

665

666

667

668 along a bed file:

669 computeMatrix reference-point $-\mathrm{S}<$ bigwig files $>-\mathrm{R}<$ bed files $>$--referencePoint center $-\mathrm{a} 1000$

670 -b 1000 -skipZeros - MAT_file.tab.gz

671

672 This is then plotted with the command:

673 plotheatmap -m MAT_file.tab.gz -out HM_file.png -colorMap Blues -missingdatacolor 1.0

675 Or, to plot the lineplots only:

676 plotProfile -m MAT_file.tab.gz -out Plot_file.png-yMax 3.0

677

678

\section{Acknowledgements}


679 The authors acknowledge the following funding sources for supporting this work. Grant \#TA-

680 RM-0614-0650-UWA from the Foundation Fighting Blindness to T.A.R., NIH NEI

681 1R01EY021482 to T.A.R., Allen Distinguished Investigator Award (Paul G. Allen Family

682 Foundation) to T.A.R., an NSF Fellowship to M.S.W. (DGE-0718124), Scholarship Wo 2010/1-

6831 from Deutsche Forschungsgemeinschaft (DFG) and the SUNY Empire Innovation Program

684 Grant to S.G.W. and NIH training grant T32HD007183 and PHS NRSA T32GM007270 from

685 NIGMS to L.S.V.

686 We thank members of the Reh and Bermingham-McDonogh laboratories for their review and

687 valuable discussion regarding the manuscript. In particular, we thank Olivia Bermingham-

688 McDonogh, Levi Todd, Marcus Hooper, and Brent Wilkerson for their comments on the

689 manuscript. Lastly, the authors thank M. Nakafuku (Cincinnati Children's) for the tetO-Ascl1-

690 ires-GFP mice.

691

692

693 Author Contributions

694 LSV and TAR conceived of all experiments and analyses. LSV conceived of and performed all 695 ATAC experiments and analyses. SGW and LSV performed FACS and RNA-seq experiments, 696 and LSV performed all analyses. MSW conceived of and performed culturing and Ascl1 ChIP697 seq experiments. KC and LC performed cell tracing and in vivo Ascll experiments and cell 698 counts. LSV and TAR wrote the manuscript with input from co-authors.

699

700

\section{References}

701 Aldiri I, Xu B, Wang L, Chen X, Hiler D, Griffiths L, Valentine M, Shirinifard A, Thiagarajan S, 
Sablauer A, et al. 2017. The Dynamic Epigenetic Landscape of the Retina During

Arnold K, Sarkar A, Yram MA, Polo JM, Bronson R, Sengupta S, Seandel M, Geijsen N, 705 Hochedlinger K. 2011. Sox2(+) adult stem and progenitor cells are important for tissue regeneration and survival of mice. Cell Stem Cell 9: 317-29.

Aydin B, Kakumanu A, Rossillo M, Moreno-Estellés M, Garipler G, Ringstad N, Flames N, subtype identities by establishing distinct chromatin landscapes. Nat Neurosci 22: 897-908.

Bringmann A, Iandiev I, Pannicke T, Wurm A, Hollborn M, Wiedemann P, Osborne NN, Reichenbach A. 2009. Cellular signaling and factors involved in Muller cell gliosis: neuroprotective and detrimental effects. Prog Retin Eye Res 28: 423-451. lineage-restricted progenitors in the mammalian retina. Development 138: 3519-31.

Buenrostro JD, Wu B, Chang HY, Greenleaf WJ. 2015. ATAC-seq: A Method for Assaying

$$
\text { Chromatin Accessibility Genome-Wide. Curr Protoc Mol Biol 109: 21.29.1-9. }
$$

\section{Casey BH, Kollipara RK, Pozo K, Johnson JE. 2018. Intrinsic DNA binding properties}

720 Castro DS, Martynoga B, Parras C, Ramesh V, Pacary E, Johnston C, Drechsel D, Lebel-Potter progenitor proliferation identified by genome-wide characterization of its targets. Genes Dev 25: 930-45.

Clark BS, Stein-O’Brien GL, Shiau F, Cannon GH, Davis-Marcisak E, Sherman T, Santiago CP, 
Hoang T V., Rajaii F, James-Esposito RE, et al. 2019. Single-Cell RNA-Seq Analysis of Retinal Development Identifies NFI Factors as Regulating Mitotic Exit and Late-Born Cell Specification. Neuron 102: 1111-1126.e5.

de Melo J, Clark BS, Blackshaw S. 2016. Multiple intrinsic factors act in concert with Lhx2 to direct retinal gliogenesis. Sci Rep 6: 32757.

Deneen B, Ho R, Lukaszewicz A, Hochstim CJ, Gronostajski RM, Anderson DJ. 2006. The Transcription Factor NFIA Controls the Onset of Gliogenesis in the Developing Spinal Cord. Neuron 52: 953-968.

Falcon S, Gentleman R. 2007. Using GOstats to test gene lists for GO term association. Bioinformatics 23: 257-258.

Fausett B V, Gumerson JD, Goldman D. 2008. The proneural basic helix-loop-helix gene asclla is required for retina regeneration. $J$ Neurosci 28: 1109-17.

Gemberling M, Bailey TJ, Hyde DR, Poss KD. 2013. The zebrafish as a model for complex tissue regeneration. Trends Genet 29: 611-620.

Goldman D. 2014. Müller glial cell reprogramming and retina regeneration. Nat Rev Neurosci 15: $431-42$.

Gordon PJ, Yun S, Clark AM, Monuki ES, Murtaugh LC, Levine EM. 2013. Lhx2 balances progenitor maintenance with neurogenic output and promotes competence state progression in the developing retina. $J$ Neurosci 33: 12197-207.

Hägglund A-C, Dahl L, Carlsson L. 2011. Lhx2 is required for patterning and expansion of a distinct progenitor cell population committed to eye development. PLoS One 6: e23387. Heinz S, Benner C, Spann N, Bertolino E, Lin YC, Laslo P, Cheng JX, Murre C, Singh H, Glass CK. 2010. Simple combinations of lineage-determining transcription factors prime cis- 
regulatory elements required for macrophage and B cell identities. Mol Cell 38: 576-89.

749

750

751

752

753

754

755

756

757

758

759

760

761

762

763

764

765

766

767

768

769

770
Heng YHE, Zhou B, Harris L, Harvey T, Smith A, Horne E, Martynoga B, Andersen J,

Achimastou A, Cato K, et al. 2015. NFIX Regulates Proliferation and Migration Within the Murine SVZ Neurogenic Niche. Cereb Cortex 25: 3758-78.

Hughes AEO, Enright JM, Myers CA, Shen SQ, Corbo JC. 2017. Cell Type-Specific

Epigenomic Analysis Reveals a Uniquely Closed Chromatin Architecture in Mouse Rod Photoreceptors. Sci Rep 7: 43184.

Jadhav AP, Roesch K, Cepko CL. 2009. Development and neurogenic potential of Müller glial cells in the vertebrate retina. Prog Retin Eye Res 28: 249-262.

Jorstad NL, Wilken MS, Grimes WN, Wohl SG, VandenBosch LS, Yoshimatsu T, Wong RO, Rieke F, Reh TA. 2017. Stimulation of functional neuronal regeneration from Müller glia in adult mice. Nature 548: 103-107.

Krueger F. 2015. Trim galore. A wrapper tool around Cutadapt and FastQC to consistently apply quality and adapter trimming to FastQ files.

Langmead B, Salzberg SL. 2012. Fast gapped-read alignment with Bowtie 2. Nat Methods 9: $357-359$.

Li H, Handsaker B, Wysoker A, Fennell T, Ruan J, Homer N, Marth G, Abecasis G, Durbin R, 1000 Genome Project Data Processing Subgroup 1000 Genome Project Data Processing. 2009. The Sequence Alignment/Map format and SAMtools. Bioinformatics 25: 2078-9.

Liao Y, Smyth GK, Shi W. 2019. The R package Rsubread is easier, faster, cheaper and better for alignment and quantification of RNA sequencing reads. Nucleic Acids Res 47: e47.

McLean CY, Bristor D, Hiller M, Clarke SL, Schaar BT, Lowe CB, Wenger AM, Bejerano G. 2010. GREAT improves functional interpretation of cis-regulatory regions. Nat Biotechnol 
28: $495-501$.

772

773

774

775

776

777

778

779

780

781

782

783

784

785

786

787

788

789

790

791

792

793

Murphy D, Hughes AEO, Lawrence KA, Myers CA, Corbo JC. 2019. Cis-regulatory basis of sister cell type divergence in the vertebrate retina. bioRxiv 648824.

Nelson BR, Ueki Y, Reardon S, Karl MO, Georgi S, Hartman BH, Lamba DA, Reh TA. 2011. Genome-wide analysis of Müller glial differentiation reveals a requirement for Notch signaling in postmitotic cells to maintain the glial fate. PLoS One 6: e22817.

Neph S, Kuehn MS, Reynolds AP, Haugen E, Thurman RE, Johnson AK, Rynes E, Maurano MT, Vierstra J, Thomas S, et al. 2012. BEDOPS: high-performance genomic feature operations. Bioinformatics 28: 1919.

Ohsawa R, Kageyama R. 2008. Regulation of retinal cell fate specification by multiple transcription factors. Brain Res 1192: 90-98.

Omori Y, Katoh K, Sato S, Muranishi Y, Chaya T, Onishi A, Minami T, Fujikado T, Furukawa T. 2011. Analysis of transcriptional regulatory pathways of photoreceptor genes by expression profiling of the Otx2-deficient retina. PLoS One 6: e19685.

Pollak J, Wilken MS, Ueki Y, Cox KE, Sullivan JM, Taylor RJ, Levine EM, Reh TA. 2013. ASCL1 reprograms mouse Müller glia into neurogenic retinal progenitors. Development 140: $2619-2631$.

Ramachandran R, Fausett B V, Goldman D. 2010. Asclla regulates Müller glia dedifferentiation and retinal regeneration through a Lin-28-dependent, let-7 microRNA signalling pathway. Nat Cell Biol 12: 1101-7.

Ramírez F, Ryan DP, Grüning B, Bhardwaj V, Kilpert F, Richter AS, Heyne S, Dündar F, Manke T. 2016. deepTools2: a next generation web server for deep-sequencing data analysis. Nucleic Acids Res 44: W160-5. 
Robinson JT, Thorvaldsdóttir H, Winckler W, Guttman M, Lander ES, Getz G, Mesirov JP. 2011. Integrative genomics viewer. Nat Biotechnol 29: 24-6.

Robinson MD, McCarthy DJ, Smyth GK. 2010. edgeR: a Bioconductor package for differential expression analysis of digital gene expression data. Bioinformatics 26: 139-40.

Shu T, Butz KG, Plachez C, Gronostajski RM, Richards LJ. 2003. Abnormal development of forebrain midline glia and commissural projections in Nfia knock-out mice. J Neurosci 23: 203-12.

Stevaux O, Dyson NJ. 2002. A revised picture of the E2F transcriptional network and RB function. Curr Opin Cell Biol 14: 684-691.

Ueki Y, Wilken MS, Cox KE, Chipman L, Jorstad N, Sternhagen K, Simic M, Ullom K, Nakafuku M, Reh TA. 2015. Transgenic expression of the proneural transcription factor Ascl1 in Müller glia stimulates retinal regeneration in young mice. Proc Natl Acad Sci 112: $13717-13722$.

VandenBosch LS, Reh TA. 2019. Epigenetics in Neuronal Regeneration. Semin Cell Dev Biol. Wilken MS, Brzezinski JA, La Torre A, Siebenthall K, Thurman R, Sabo P, Sandstrom RS, Vierstra J, Canfield TK, Hansen R, et al. 2015. DNase I hypersensitivity analysis of the mouse brain and retina identifies region-specific regulatory elements. Epigenetics Chromatin 8: 8 .

Wohl SG, Reh TA. 2016. The microRNA expression profile of mouse Müller glia in vivo and in vitro. Sci Rep 6: 35423 .

Wu L, Timmers C, Maiti B, Saavedra HI, Sang L, Chong GT, Nuckolls F, Giangrande P, Wright FA, Field SJ, et al. 2001. The E2F1-3 transcription factors are essential for cellular proliferation. Nature 414: 457-462. 
817 Young RW. 1985. Cell differentiation in the retina of the mouse. Anat Rec 212: 199-205.

818 Zibetti C, Liu S, Wan J, Qian J, Blackshaw S. 2019. Epigenomic profiling of retinal progenitors reveals LHX2 is required for developmental regulation of open chromatin. Commun Biol 2:

820 142.

821

822

823

824

825

826

Figure 1. Progenitor and Müller Glial accessibility is generally similar.

827 A. ATAC experimental design. B-C. Genomic tracks of P2 progenitor ATAC and whole retina

828

DNase(Wilken et al. 2015) around Ascll and Dll1. D. Tracks for progenitors and adult MG

829 (Jorstad et al. 2017) ATAC-seq at glial genes and E. progenitor genes. F. BEDOPS overlaps of

830 progenitor and adult MG peak calls. F'. Genomic region annotation of accessibility profiles. F'.

831 Tag density in progenitor and adult MG ATAC at accessibility profiles.

832

833

834

835 Figure 2. Cis-regulatory binding motifs differ in Müller glia development

836 A-C. Gene ontology associated with accessibility changes (A. P2-adult decreasing, B. P2-Adult

837 increasing, C. P8-Adult decreasing,). D-F. Predicted motif enrichment for accessibility changes

838 (D. P2-Adult decreasing [bedops], E. P2-Adult increasing [bedops], F. P2-P8 increasing [DA]).

839 G-I. Central enrichment of motifs for progenitor- and adult MG-specific accessibility regions. J. 
840 Co-occurrence analysis for top predicted motifs in progenitor-specific accessibility regions

841 clustered by co-occurrence ratios.

842

843

844

845

846

847

848

849

850

851

852

853

854

855

856

857

858

859

860 A. Cell tracing of progenitors and MG was induced with CC-GFP or Ascl1-ires-GFP on a Glast

861 promoter at P0, P4, P8/9, and P12. All analyzed at P21. Scale bar 50 um B. Graphical summary

862 of reprogramming observed with Ascl1. C. Cell counting quantification of GFP cell tracing in

Figure 3. Glial expression and accessibility profiles follow developmental trends
A. Experimental design for FACS isolation and RNA-seq of developing MG. B. MA plot of

expression from progenitors to adult MG demonstrating thresholds for filtering high fold change

and counts for the top genes with changing expression. C. Overlaps of top gene expression

changes with ATAC profiles. The percentage of genes that increase or decrease expression are

represented on the y axis, split by ATAC categories(x). D. Euclidian hierarchical clustering by

expression changes from P2 to Adult, heat map of top gene expression, and related gene

ontology categories. Categories marked with a * indicates categories that overlap with ATAC

MG increased accessibility. Those marked with a ** indicate where progenitor-specific

accessibility overlaps, and $* * *$ indicates where shared accessibility overlaps. E. Motif

enrichment for accessible regions with gained expression and accessibility (GOE GOA), lost

expression and accessibility (LOE LOA), and lost expression and shared accessibility (LOE SA).

F-G. Expression profiles of (F) progenitor genes of interest, and (G) NFI transcription factors.

Figure 4. Ascll overexpression at young ages is sufficient to generate bipolar neurons 
863 (A). D. Cell counting quantification of Ascll induction tracing in (A).

864

865

866 Figure 5. Ascl1 differentiates Bipolar fates from Rods

867 A. Analytical design comparing the accessible regions in neurons((Hughes et al. 2017; Jorstad et 868 al. 2017) with ATAC-seq from progenitors and MG. B, D. Rod chromatin accessibility overlaps 869 with both progenitor(B) and adult MG(D) accessibility by $19 \mathrm{k}$ regions. C,E. Otx2 predicted 870 central enrichment is high in Rod-specific accessible regions, and low in regions shared with 871 progenitors (C) and adult MG (E). F,G. Predicted motifs for rod-specific (F) and Bipolar-specific

872 (G) accessible regions. H. Experimental design for Ascl1 ChIP-seq. I. Ascl1 ChIP-seq read

873 density in Rod, Bipolar, and Progenitor accessible regions. J,K. Central enrichment for Ascl1 874 binding in rod, bipolar, and progenitor $(\mathrm{J})$ or adult $\mathrm{MG}(\mathrm{K})$ accessible regions. 
bioRxiv preprint doi: https://doi.org/10.1101/752428; this version posted September 3, 2019. The copyright holder for this preprint (which was not certified by peer review) is the author/funder. All rights reserved. No reuse allowed without permission.

\section{A}

Sox2-GFP
Rlbp-CreER;TdTomato
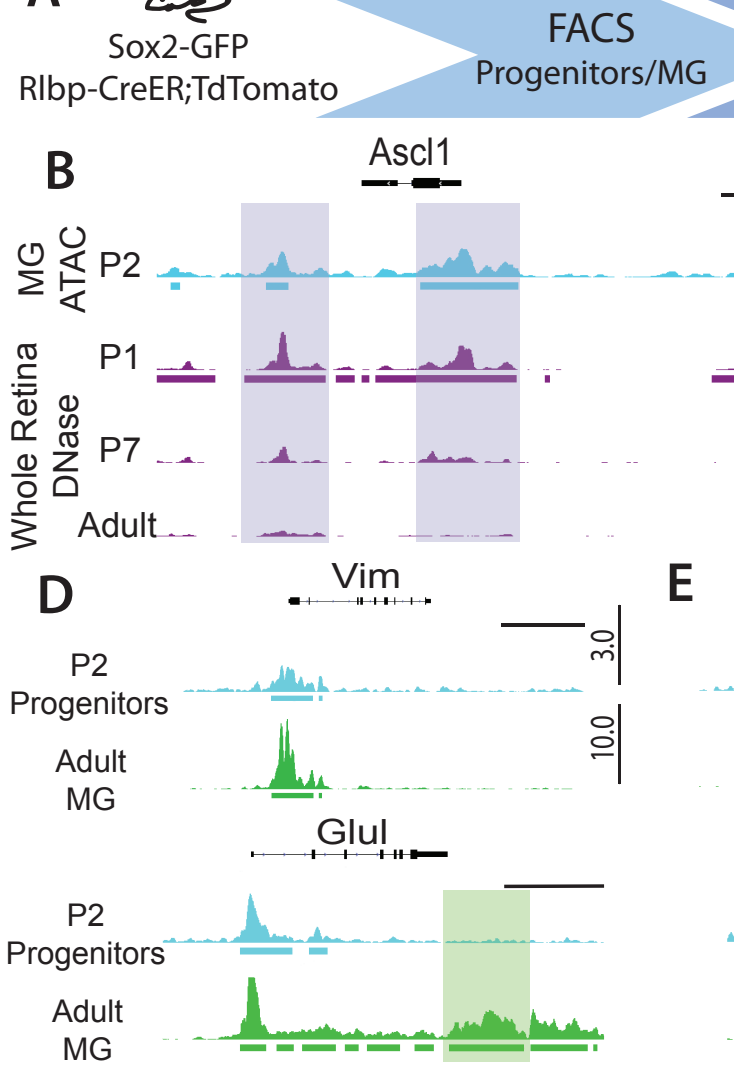
bioRxiv preprint doi: https://doi.org/10.1101/752428; this version posted September 3, 2019. The copyright holder for this preprint (which was not certified by peer review) is the author/funder. All rights reserved. No reuse allowed without permission.

\section{A P2-Adult $\downarrow$}

Nervous System Development

Multicellular Organism Development

Developmental Process

Anatomical Structure Development

System Development

Anatomical Str Morphogenesis

Neurogenesis

Cell Differentiation

Cellular Developmental Process

Generation of Neurons

- $\log 10$ (Pvalue)

B P2-Adult $\uparrow$

Cellular Process

Biological Regulation

Cellular Metabolic Process

Primary Metabolic Process

Cell Macro Metabolic Process

Cell Component Biogenesis

N-Compound Metabolic Proc.

Organic Metabolic Process

Reg. of Biological Process

Cell Component Organization - $\log 10$ (Pvalue)

C

P8-Adult $\downarrow$

Cellular Process

Nervous System Development

Reg. Neuron Differentiation

Neuron Differentiation

Generation of Neurons

Negative Regulation of Cell Process

Cellular Metabolic Proccess

Neg. Regulation Biological Proccess

Macromolecule Metabolic Process

Developmental Process
D P2-Adult $\downarrow$

\begin{tabular}{|c|c|}
\hline Motif Logo & Class \\
\hline CCACTAGGGGGC & CTCF \\
\hline 술술도IAATTA술 & $\begin{array}{c}\text { Homeobox } \\
(\text { (Lhx) }\end{array}$ \\
\hline AGCAGATGGC & \begin{tabular}{|c|} 
bHLH \\
(NeuroD1)
\end{tabular} \\
\hline C्CATTTGTTT & HMG (Sox) \\
\hline CAGGACAG & \begin{tabular}{|c|} 
Nuclear \\
Receptor
\end{tabular} \\
\hline
\end{tabular}

40

E

P2-Adult $\uparrow$

\begin{tabular}{|c|c|}
\hline Motif Logo & Class \\
\hline GCCСТСТАGTGG & CTCF \\
\hline 두ㅅㅜㅗAATIA & $\begin{array}{c}\text { Homeobox } \\
(\text { (Lhx) }\end{array}$ \\
\hline 둣두섣CAA & $\begin{array}{l}\mathrm{NF} \\
(\mathrm{NFIX}) \\
\end{array}$ \\
\hline AACTAGGTCA & RORgt \\
\hline AGCGCCACPCT & $\begin{array}{l}\text { Zinc Finger } \\
\text { (CTCFL) }\end{array}$ \\
\hline
\end{tabular}

$\mathbf{F}$

\begin{tabular}{|c|c|}
\hline Motif Logo & Class \\
\hline CACTAGATGGCA & CTCF \\
\hline 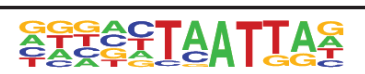 & $\begin{array}{c}\text { Homeobox } \\
\text { (Lhx) }\end{array}$ \\
\hline GATCACAGCCAA & \begin{tabular}{|c}
$\begin{array}{c}\text { Zinc Finger } \\
(\mathrm{GFI})\end{array}$ \\
\end{tabular} \\
\hline CCCANTTGT & HMG (Sox) \\
\hline AGCITTCCCA AT & $\mathrm{NFI}$ \\
\hline
\end{tabular}

G

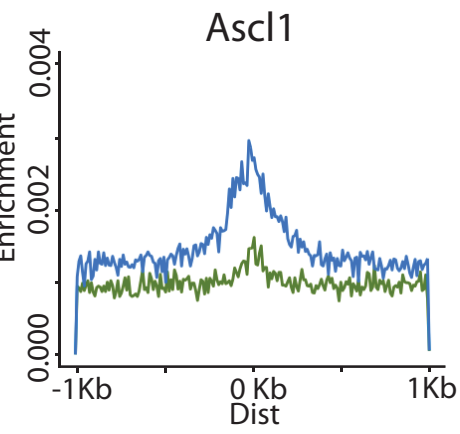

$\stackrel{ \pm}{ \pm}$-Adult MG

H $\stackrel{\oplus}{\sim}$-Progenitor

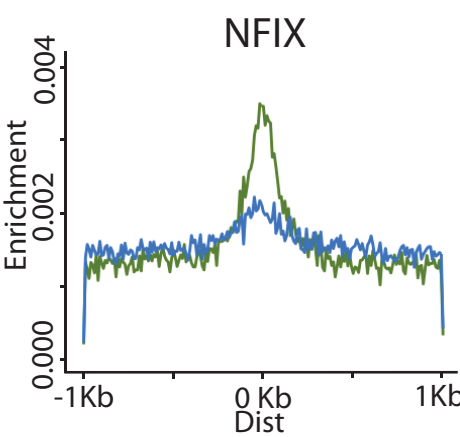

I

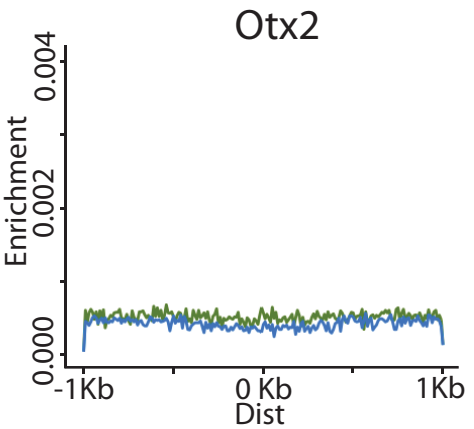

$\begin{array}{lr}10 & 15 \\ -\log 10(\text { Pvalue) }\end{array}$

20

- $\log 10($ Pvalue)

J

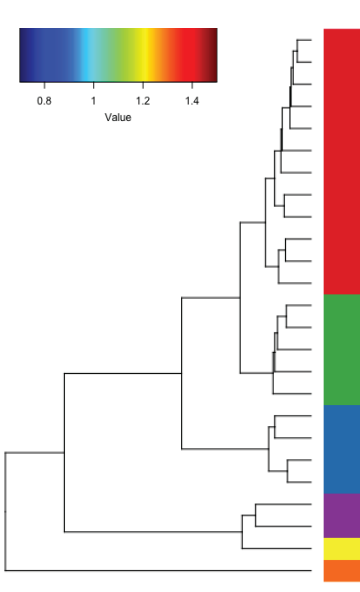

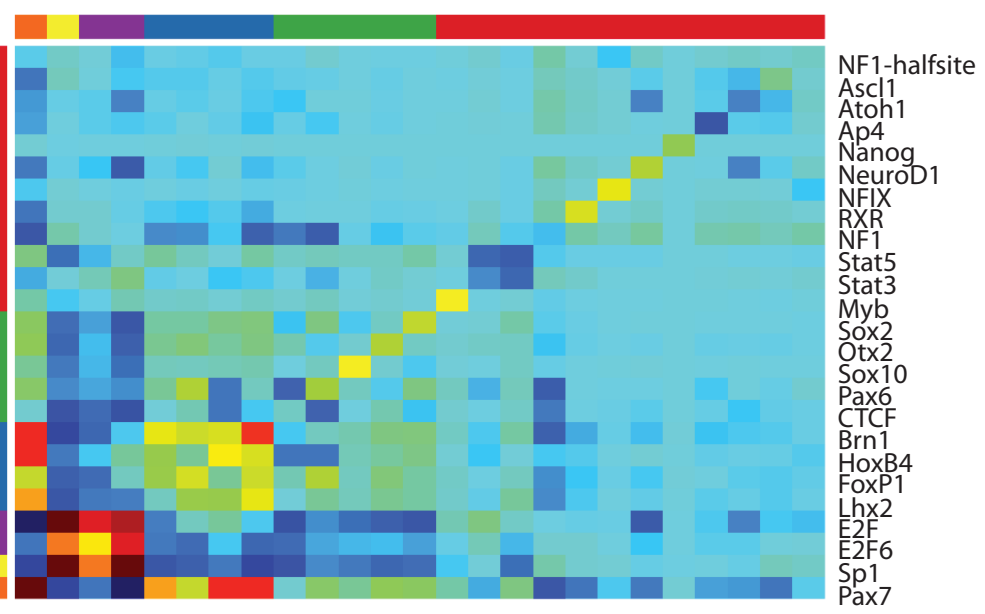

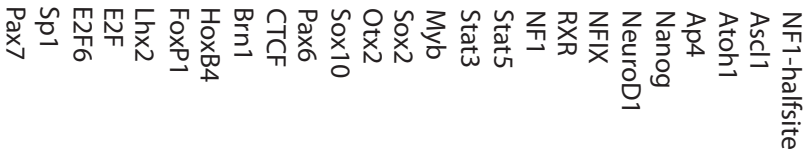


bioRxiv preprint doi: https://doi.org/10.1101/752428; this version posted September 3, 2019. The copyright holder for this preprint (which was

A not certified by peer review) is the author/funder. All rights reserved. No reuse allowed without permission.

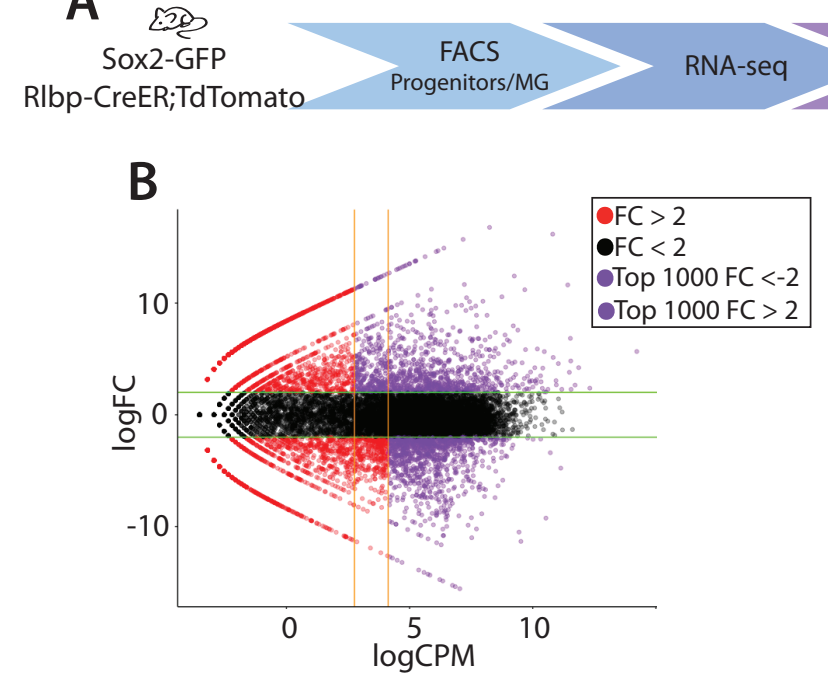

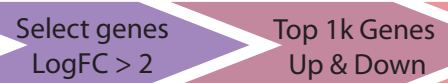

C

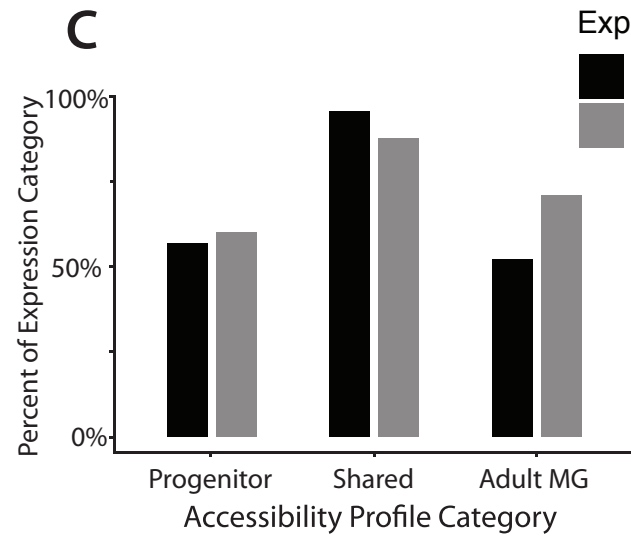

Overlap ATAC genes Increasing

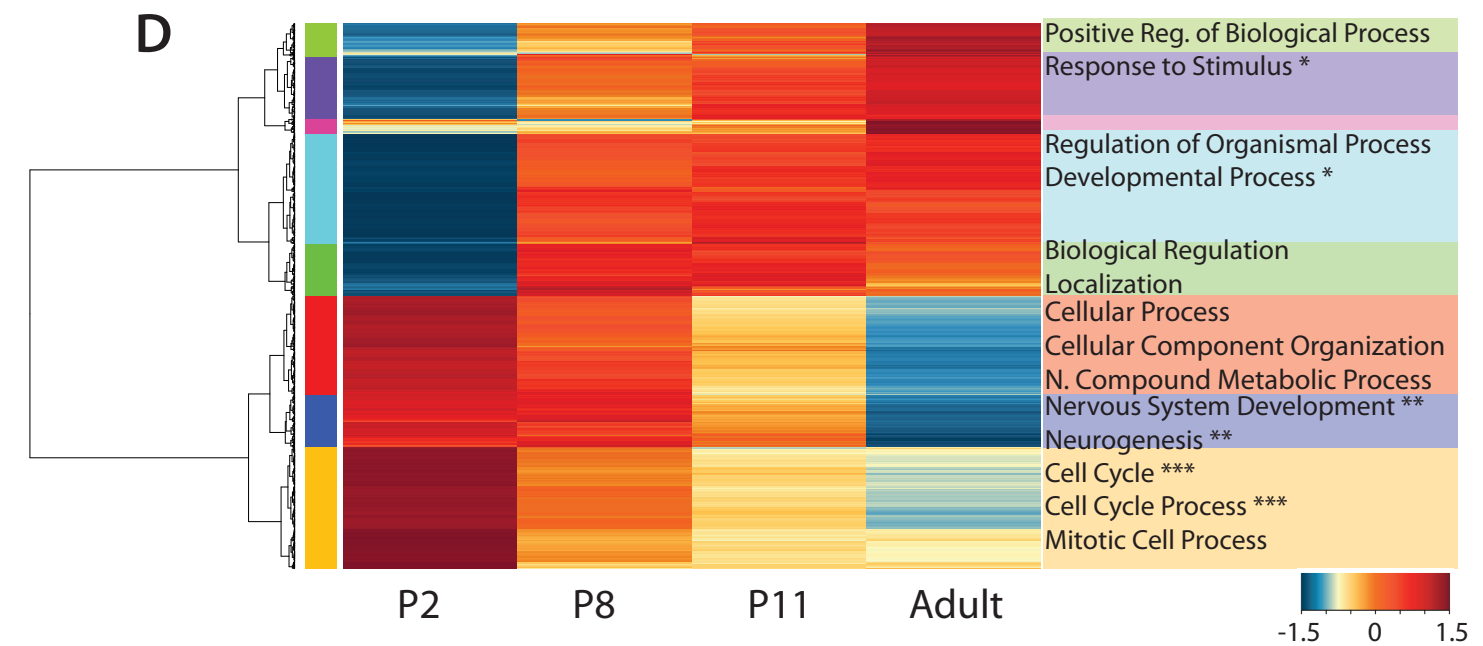

E

\begin{tabular}{|c|c|c|}
\hline & -ogo & lass \\
\hline & & Cic \\
\hline & & Lhx \\
\hline & & NFIX \\
\hline & TGACCT & RORgt \\
\hline & ETIAO & NFIL \\
\hline
\end{tabular}

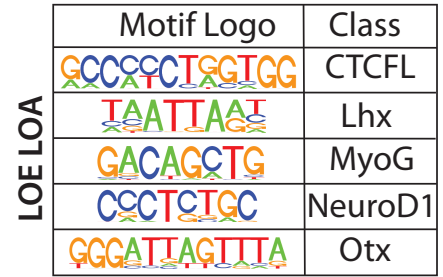

\begin{tabular}{|c|c|c|}
\hline & Motif Logo & Class \\
\hline & SCCATCTTAGTGG & CTCF \\
\hline & 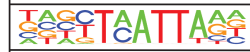 & Lhx \\
\hline & CGAACAAAGG & Sox \\
\hline & ICGCGCCAAA & $\mathrm{E} 2 \mathrm{~F}$ \\
\hline & 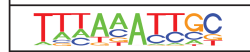 & Lin54 \\
\hline
\end{tabular}

F

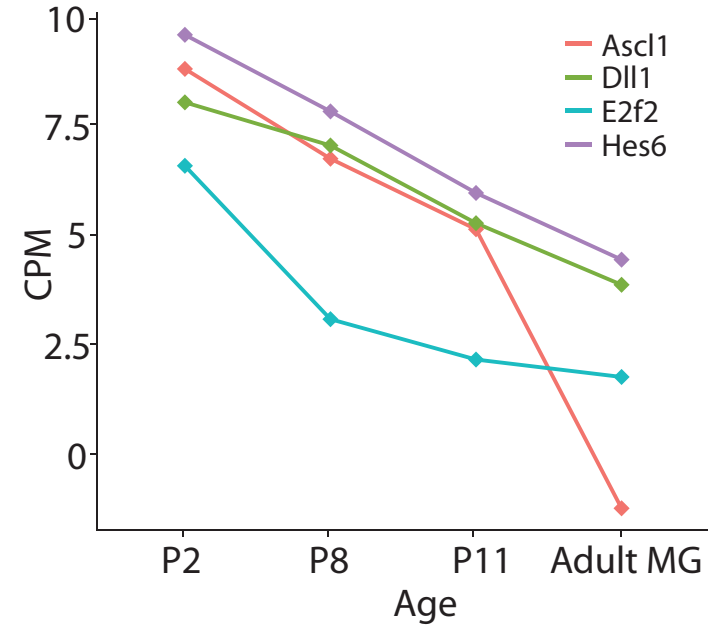

G

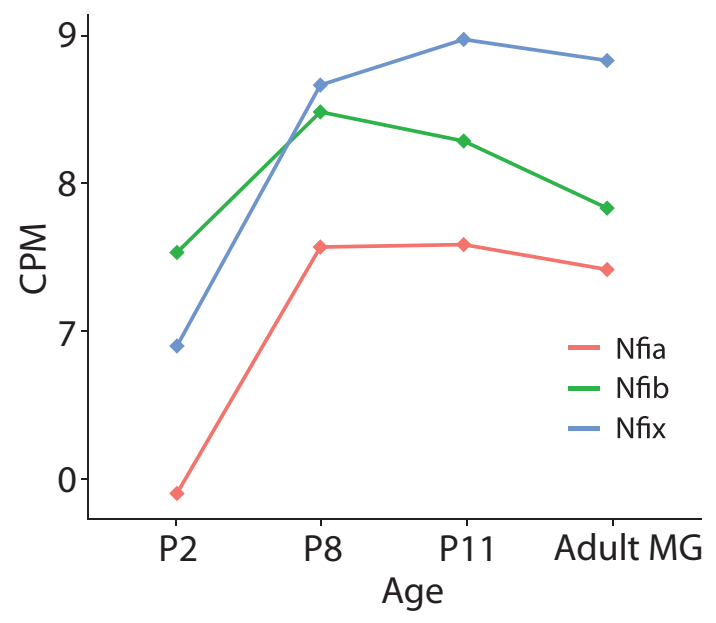


bioRxiv preprint doi: https://doi.org/10.1101/752428; this version posted September 3, 2019. The copyright holder for this preprint (which was not certified by peer review) is the author/funder. All rights reserved. No reuse allowed without permission.

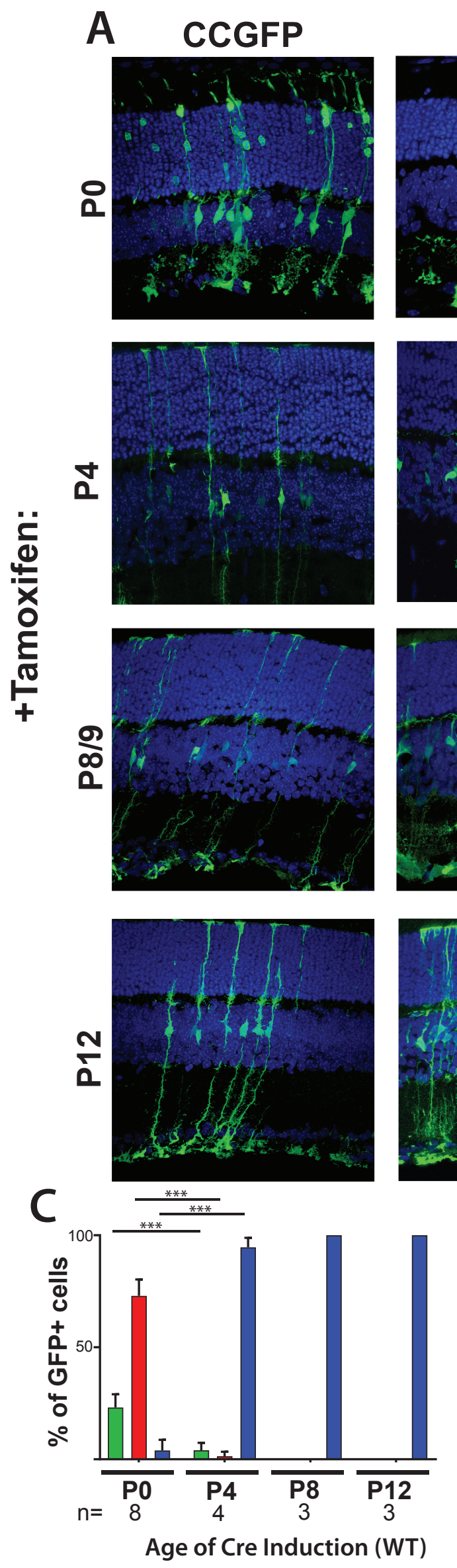

Ascl1
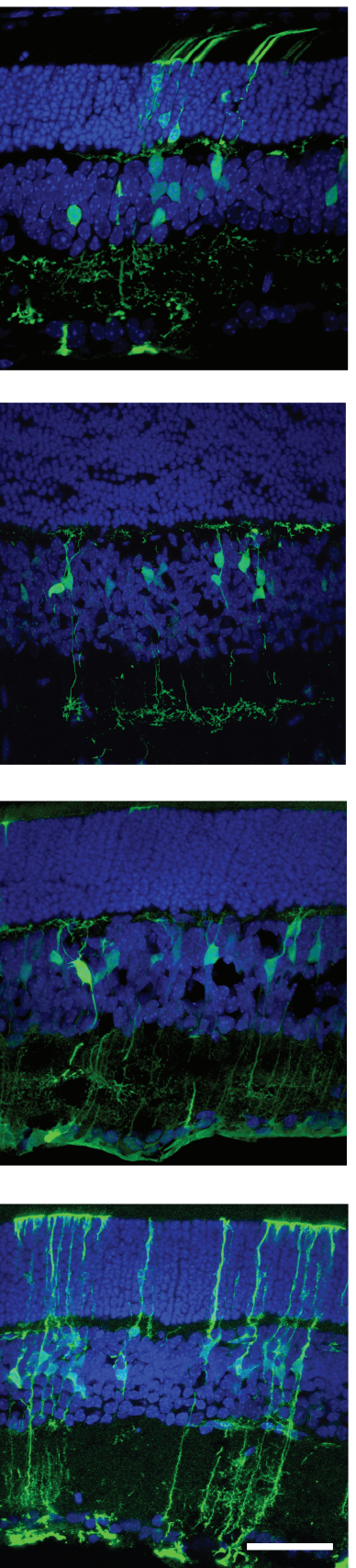

B

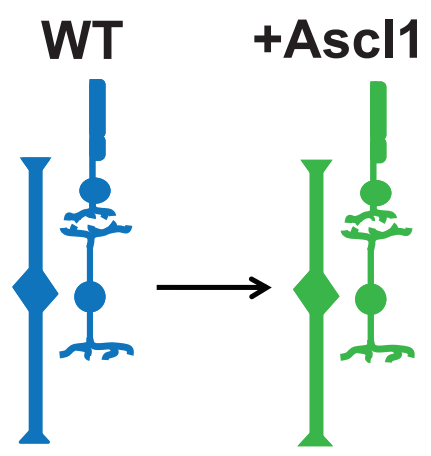

T1
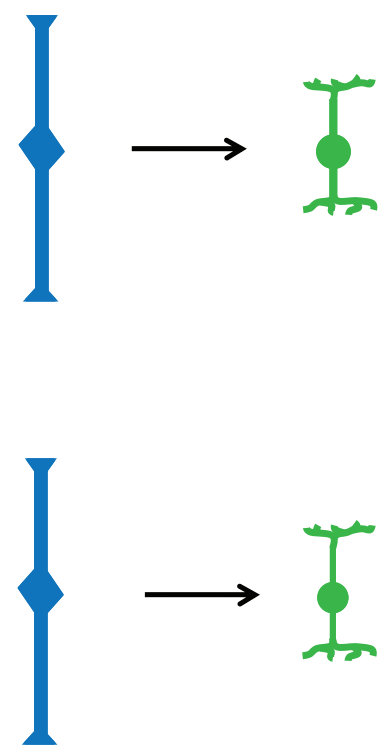

T2

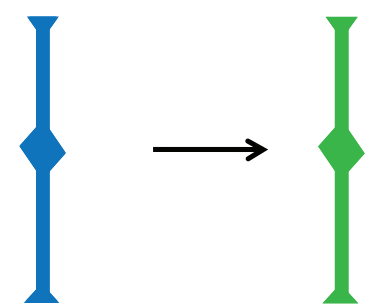

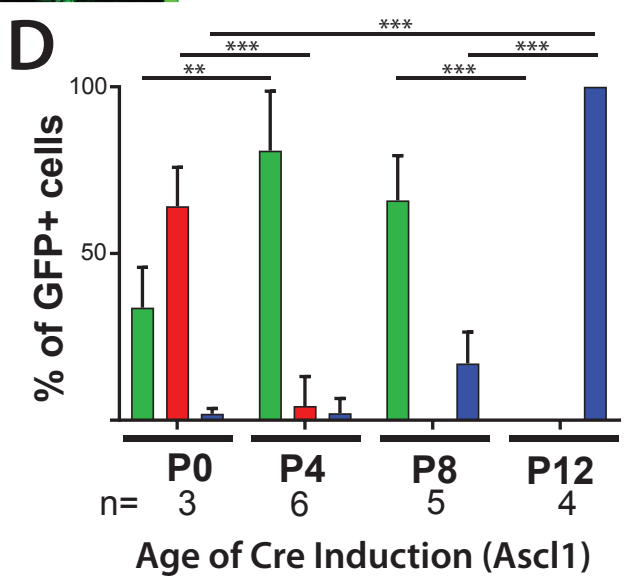

Bipolar

Photoreceptor

Müller Glia 
bioRxiv preprint doi: https://doi.org/10.1101/752428; this version posted September 3, 2019. The copyright holder for this preprint (which was not certified by peer review) is the author/funder. All rights reserved. No reuse allowed without permission.

A ATAC Peaks:

Rod
Bipolar
Progenitor
Adult MG
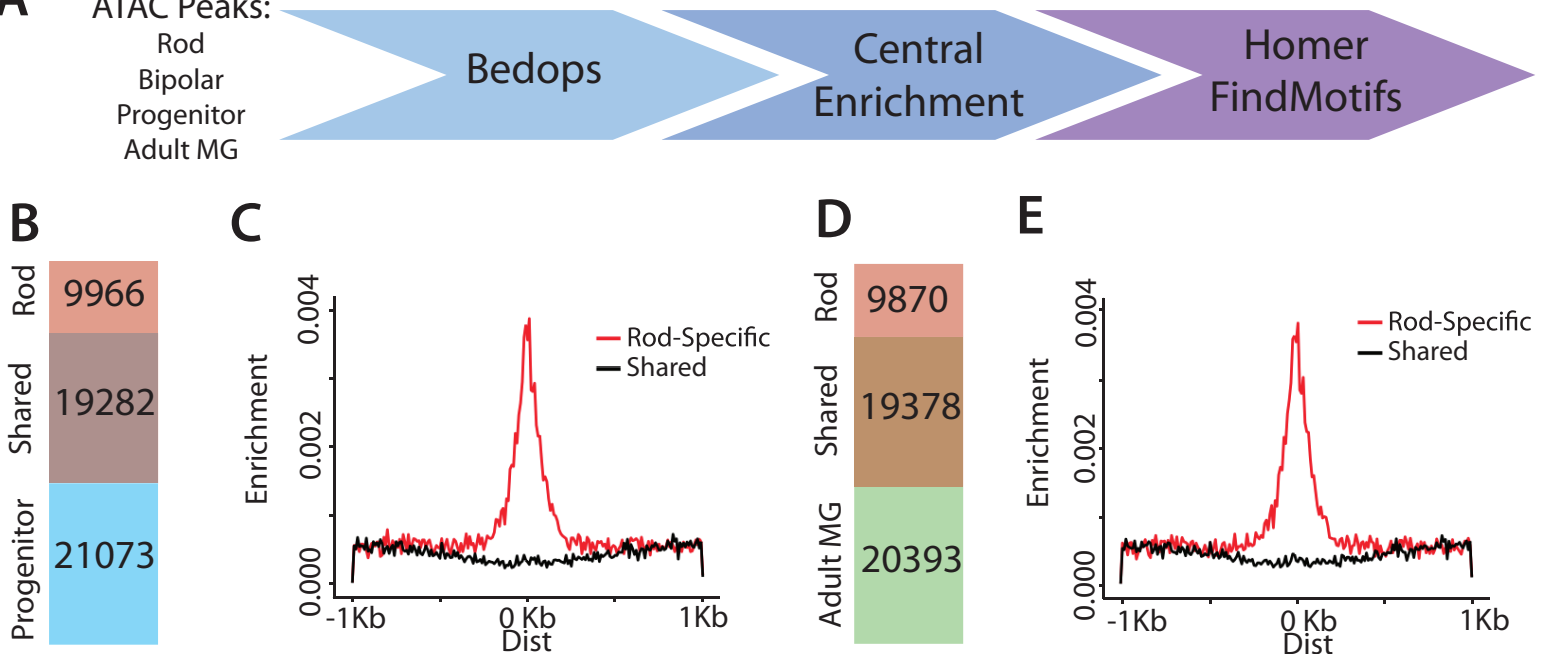

C

D $\quad E$
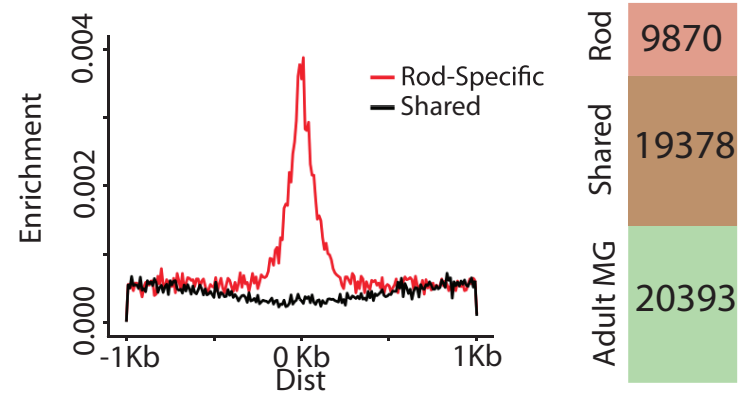

E

F

Rod Motifs

G

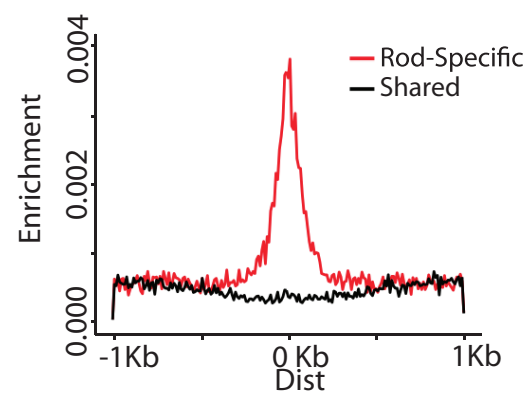

\begin{tabular}{|c|c|}
\multicolumn{2}{c}{ Rod Motifs } \\
\hline Motif Logo & Class \\
\hline TAATCCTCTT & Homeobox \\
\hline CCACCAGGAGGGC & CTCFL \\
\hline TAATTAGC & Lhx \\
\hline
\end{tabular}

H

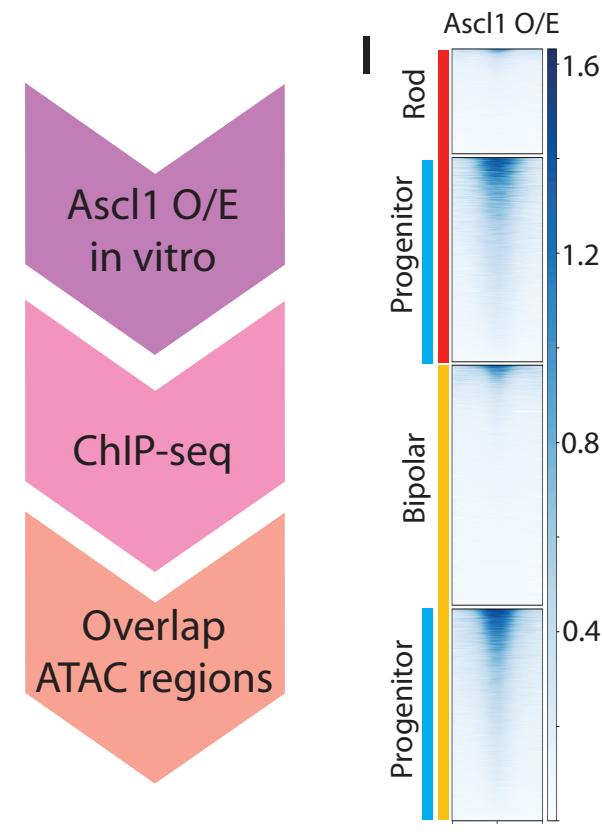

\begin{tabular}{|c|c|}
\hline Motif Logo & Class \\
\hline AA술션GATTA & Homeobox \\
\hline GCCATCTGGTGG & CTCF \\
\hline 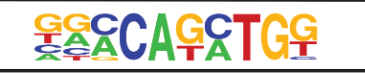 & $\mathrm{bHLH}$ \\
\hline
\end{tabular}
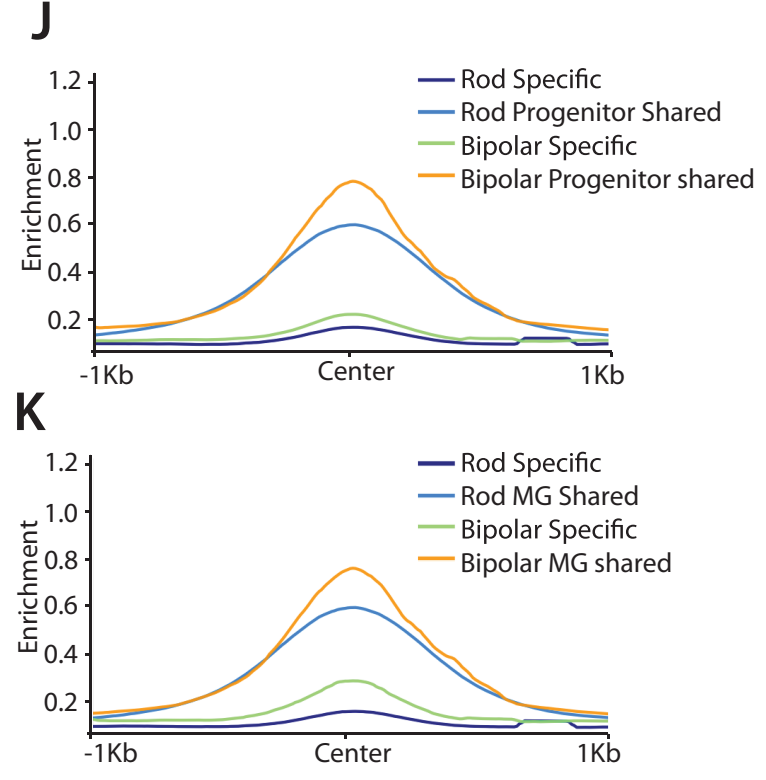